\title{
Visualization Of Thermal Distribution During Machining of Dental Implants
}

\author{
Apurba Kumar Roy ${ }^{1}$ (D), Kaushik Kumar ${ }^{1, *}$ (D) \\ 1 Dept. of Mechanical Engg., Birla Institute of Technology, Mesra; akroy@bitmesra.ac.in_(A.K.R.); kkumar@bitmesra.ac.in \\ (K.K.); \\ * Correspondence: kkumar@bitmesra.ac.in (K.K.);
}

Scopus Author ID 8972729400

Received: 12.08.2021; Revised: 25.09.2021; Accepted: 29.09.2021; Published: 16.10.2021

\begin{abstract}
Dental implants used are usually metallic. One of the most widely used materials for the same is Titanium-based alloy like Ti-6Al-4V, which suffers difficulty processing and machining due to its thermo-physical properties. The thermo-physical property of the material plays a significant role in the biocompatibility and safety to use them as dental implants. Due to its hardness and difficult-tomachine characteristics, a large amount of heat gets generated while machining, creating dimensional error. Hence before assembly of parts, they must be processed so that stress deformation of the assembly due to heat can be avoided. During machining of Ti-6Al-4V, the cooling strategy needs prior information on the thermal field, and hence, the distribution of temperature in the material is an essential domain to study. To understand the thermal distribution in the material during machining, 3dimensional heat diffusion equations have been solved using a Finite Difference scheme coupled with the Liebmann method to generate the thermal distribution in the material. An efficient parallelized code for the same has been written in MATLAB and utilized in this numerical study. This study reveals the variation of the temperature gradient with time and space, all along with the three orthogonal directions, which will be helpful for the scientists, engineers, and surgeons to ascertain the sustainability $[1,2]$, suitability, and longevity of the implants.
\end{abstract}

Keywords: Dental Implants; Thermal Distribution in Machining; Numerical Schemes; 3D Heat Diffusion; Transient Heat Diffusion; Finite-Difference method; Liebmann method; Matlab, Vectorized Cloud Computing.

(C) 2021 by the authors. This article is an open-access article distributed under the terms and conditions of the Creative Commons Attribution (CC BY) license (https://creativecommons.org/licenses/by/4.0/).

\section{Introduction}

The dental implant, medically termed an endosseous implant, is a part used as a bridge between the bone of the jaw or skull and the artificial denture. These are metallic (the most common one is a titanium alloy Ti-6Al-4V) and usually comprise three sections, as shown in Figure 1 (a) [3], out of which two are metallic. The lower part (implant) goes into the jaw and the upper part (abutment) within the crown [4]. As the implant part is required to be fitted inside the human body, machining requires precision, dimensional stability, and accuracy [5]. The problem gets more complicated as the two parts need a different type of machining status. The implant is roughly machined (for coating purposes), whereas the abutment requires fine machining (Figure 1 (b) [6]). Ti-6Al-4V owing to its hardness and difficult to machine characteristics, generates a large amount of heat which creates dimensional error while machining. Hence prior to assembly of parts, they are required to be processed so that stress deformation of the assembly due to heat can be avoided. Moreover, the cooling strategy $[7,8]$ 
needs prior information on the thermal field, and hence, the distribution of temperature in the material is an essential domain to study. The present paper focuses on the same.

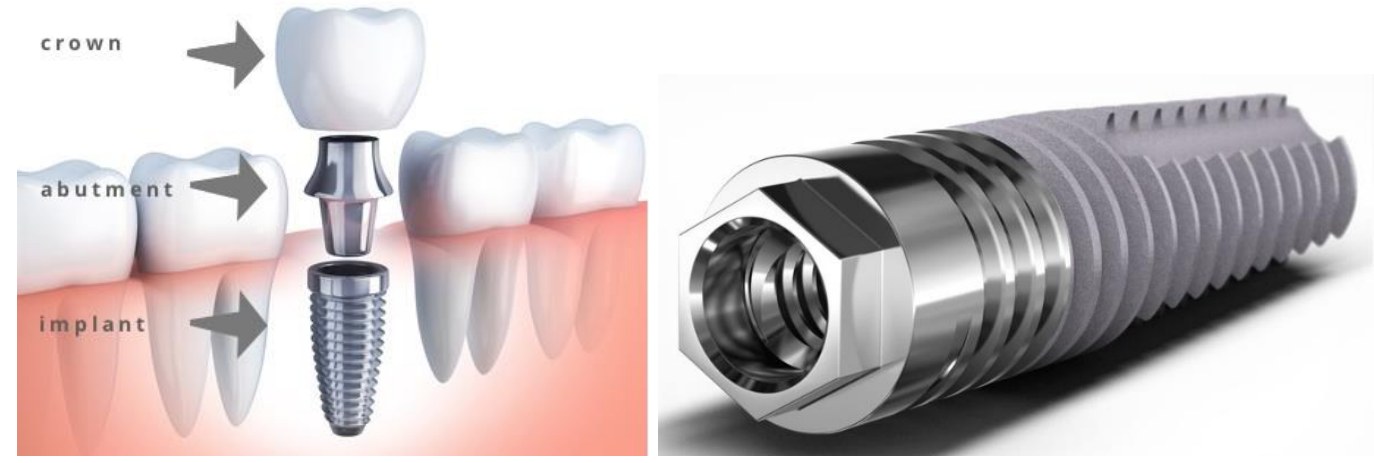

Figure 1. Dental implants: (a) components [3]; (b) machining conditions [6].

The heat conduction process involves the flow of heat energy from a higher thermal gradient zone to a lower thermal gradient zone through materials. Thermal systems having pseudo transient phase, after the passage of time reaches the steady-state situation. A complex mathematical treatment coupled with Taylor series expansion is required to solve steady-state or transient heat conduction/diffusion problems [9, 10]. Scientists and engineers extensively utilize various numerical schemes to solve real-life problems with various stringent boundary conditions [11-14]. Shen et al. [15] highlighted the benefit of various numerical schemes for numerical analysis of thermal flow field. Karaa and Zhang [16] discussed the advantages and limitations of various iterative schemes to solve conduction/ convection problems. Mishra [17] carried out the numerical heat transfer analysis through a 2-dimensional plate with various popular numerical schemes. Hard materials like titanium and its alloys, which are extensively used in implants, have different thermo-physical properties as compared to industrial/structural materials. The processing of such metal and alloys play a crucial role in its usability [18]. Heat flow analysis through absorbing medium has been made by Kovtanyuk and Chebotarev [19]. Lam [20], Lam, and Fong [21] utilized the Phase Lagging technique to solve various heat diffusion problems. Woodbury et al. [22] developed an analytical solution for 2-dimensional heat diffusion through a thin plate with constrained heating at one of its boundaries. Miranda and Felipe [23] worked on diagnosing cancerous and malignant cells in the human body utilizing various heat sources. Similarly, Frunza and Luca [24] did for laser-assisted surgery and dental contacts. Computational studies on heat transfer in electronic microchips for complex network circuits have been carried out by Bahşi and Çevik [25]. The estimation of the distribution of temperature for bi-directional unsteady heat diffusion utilizing finite difference method [26], a multi-grid technique[27], heat networks[28] in a steady-state condition and moving source technique [29], heating and cooling of 2-dimensional solid using CattaneoVernotte technique for realistic analysis [30], computational analysis on heat conduction using Monte-Carlo technique [31] and process of verification of code [32] helped in basic understanding the aspects of numerical heat conduction/diffusion in different engineering materials $[33,34]$.

Reviewing the related research papers in the conduction/diffusion of heat, it can be seen that transient analysis of multi-dimensional heat flow characteristics is scanty, and the optimal solution of such complex engineering problems poses a numerical challenge. The useful solution of multi-dimensional heat transfer requires an efficient computational algorithm coupled with computational resources, which otherwise incur computational time and cost. 
The present work relates to numerical analysis on a 3-dimensional transient heat conduction/diffusion problem using the most popular Finite Difference scheme. In this analysis, the effects of radiation and advection have been neglected. An efficient parallelized code has been written in MATLAB (R-2021a), and code validation has been done with the analytical solution.

\section{Materials and Methods}

\subsection{Model equations and statement of the problem.}

The flow of heat energy through a solid material from a higher temperature to a lower temperature zone in multiple directions is governed by the law of Fourier heat conduction [35]. The general form of governing heat equation for the case of transient heat conduction/diffusion can be written as [36],

$$
\rho c_{p} \frac{\partial u_{(r, t)}^{\mathrm{r}}}{\partial t}=P \nabla^{2} u(\stackrel{\mathrm{r}}{u}, t)
$$

where $\mathrm{u}$ is the field variable, and it is equivalent to temperature. $\rho$ is the density of the material. $c_{p}$ being the specific heat and $P$ is the thermal conductivity of the material. The space vector

$r=(x, y, z)$

The gradient term in the right hand side in the equation (1) is expressed as

$$
\nabla=\frac{\partial}{\partial x} \underset{i}{i}+\frac{\partial}{\partial x} \underset{j}{j}+\frac{\partial}{\partial x} \stackrel{\mathrm{r}}{k}
$$

In the case of constant thermo-physical property of the material, the equation (1) can be expressed as

$$
\frac{\partial u^{1}(r, t)}{\partial t}=\nabla \cdot\left(\left[\frac{P}{\rho c_{p}}\right] \nabla u(r, t)\right)
$$

Let $c_{p}$ is written as $c, P / \rho c_{p}=\varphi, \quad \stackrel{1}{u}(r, t)=u$

the equation (4) reduces to

$$
\frac{\partial u}{\partial t}=\nabla \cdot([\varphi] \nabla u)
$$

The term ' $\varphi$ ', the thermal diffusivity, plays a crucial role in the heat transfer through conducting material. To obtain the solution of heat equation as shown in equation (5), which is exhaustive and the solution schemes are based on some crucial factors such as the geometrical domain of the heat flow, the complexity of the boundary conditions, and desired numerical accuracy desired in the solution. One of the most accurate ways to get the thermal field solution within the material is to adapt the analytical solution technique. To formulate the analytical expression for the three dimensional heat flow, the technique of separation of a variable can be used, which is explained below. Rewriting the equation(5) for three dimensional Cartesian coordinate, the following relation can be obtained

$$
\frac{\partial u}{\partial t}=\varphi\left(\frac{\partial^{2} u}{\partial x^{2}}+\frac{\partial^{2} u}{\partial y^{2}}+\frac{\partial^{2} u}{\partial z^{2}}\right)
$$


Using the separation of variable method, let us express the temperature $\mathrm{u}$ as $u(x, y, z, t)=X(x) Y(y) Z(z) \tau(\mathrm{t})$

Substituting the equation(7) into equation(6), which results in

$\frac{1}{X} \frac{d^{2} X}{d X^{2}}+\frac{1}{Y} \frac{d^{2} Y}{d Y^{2}}+\frac{1}{Z} \frac{d^{2} Z}{d Z^{2}}=\frac{1}{\varphi \tau} \frac{d \tau}{d t}=-\mu^{2}$

where $-\mu^{2}$ is the separation constant, and it is a real number. To obtain bounded solution, it is required to separate the function of time, which is $\tau$, to the right-hand side from X, Y, Z. From the

expression of $\frac{1}{\varphi \tau} \frac{d \tau}{d t}$ in equation(8), the solution becomes

$$
\tau(t)=G e^{-\varphi \mu^{2} t}
$$

where $\mathrm{G}$ is the integrating constant. The next step is to separate the expression of $\mathrm{X}, \mathrm{Y}$ from $\mathrm{Z}$. for this, taking $\frac{1}{Z} \frac{d^{2} Z}{d Z^{2}}$ portion in equation (8) on the right-hand side and assuming another separating constant $-\lambda^{2}$, it is possible to write the following equation as shown in equation(10).

$$
\frac{1}{X} \frac{d^{2} X}{d X^{2}}+\frac{1}{Y} \frac{d^{2} Y}{d Y^{2}}=-\frac{1}{Z} \frac{d^{2} Z}{d Z^{2}}-\mu^{2}=-\lambda^{2}
$$

Hence the $\mathrm{Z}$ equation becomes

$$
\frac{d^{2} Z}{d Z^{2}}-\gamma^{2} Z=0
$$

where $\gamma^{2}=\mu^{2}-\lambda^{2}$. Thus the solution of $\mathrm{Z}$ equation becomes

$$
Z(z)=E_{1} \cos \gamma z+F_{1} \sin \gamma z
$$

The next step is to separate out the function $\mathrm{X}$ and $\mathrm{Y}$ as follows:

$$
\frac{1}{X} \frac{d^{2} X}{d X^{2}}=-\frac{1}{Y} \frac{d^{2} Y^{2}}{d Y^{2}}-\lambda^{2}=-\alpha^{2}
$$

Here $-\alpha^{2}$ is another separation constant and hence the following relations can be obtained.

$$
\begin{aligned}
& \frac{d^{2} X}{d X^{2}}+\alpha^{2} X=0 \\
& \frac{d^{2} Y^{2}}{d Y^{2}}+\beta^{2} Y=0
\end{aligned}
$$

where $\beta^{2}=\lambda^{2}-\alpha^{2}$.

Now the solution for the $\mathrm{X}$ is obtained as

$X(x)=A \cos \alpha x+B \sin \alpha x$

Similarly for $\mathrm{Y}$, it is written as

$$
Y(y)=C \cos \beta y+D \sin \beta y
$$

Therefore, the required relation is

$u(x, y, z, t)=(\mathrm{A} \cos \alpha x+B \sin \alpha x)(C \cos \beta y+D \sin \beta y)\left(E_{1} \cos \gamma z+F_{1} \sin \gamma z\right) \mathrm{e}^{-\varphi \mu^{2} t}$ 
In equation(18), the constants have been redefined as $E=E_{l} G, \quad F=F_{l} G$ and $\mu^{2}=\alpha^{2}+\beta^{2}+\gamma^{2}$

To obtain the solution in equation(18), using $\mathrm{A}=\mathrm{B}=\mathrm{C}=\mathrm{D}=\mathrm{E}=\mathrm{F}=1$ and taking a parallelepiped of characteristic dimensions $0 \leq x \leq a, 0 \leq y \leq b, 0 \leq z \leq c$ and which are kept at zero temperature.

If initially, the temperature of the solid is given by

$u(x, y, z, t=0)=f(x, y, z)$

having the boundary conditions(BCs)

$u(0, y, z)=u(x, 0, z)=u(x, y, 0)=0$

The final solution is given in equation(21)

$u(x, y, z, t)=\frac{8}{a b c} \sum_{m=1}^{\infty} \sum_{n=1}^{\infty} \sum_{q=1}^{\infty} F(m, n, q) \sin \left(\frac{m \pi x}{a}\right) \sin \left(\frac{n \pi y}{b}\right) \sin \left(\frac{q \pi z}{c}\right) e^{-\lambda^{2} t}$

where

$F(m, n, q)=\int_{0}^{a} \int_{0}^{b} \int_{0}^{c} f(x, y, z) * \sin \left(\frac{m \pi x}{a}\right) \sin \left(\frac{n \pi x}{b}\right) \sin \left(\frac{q \pi x}{c}\right) \mathrm{dxdydx}$

Hence, equation (21) gives the analytical solution for the 3D transient heat equation with specified initial and boundary conditions [37].

Equation (21) is the benchmark for comparing the 3D transient heat equation solution using various methods. Although equation (21) is supposed to fetch the best and accurate solution, obtaining the thermal flow field using the analytical relation mentioned in equation(21) is indeed a herculean task requiring further mathematical treatment. Hence a compatible numerical technique has been adopted for the solution in the heat equation(6).

Rewriting the equation(6) as

$$
\left(\frac{\partial^{2} u}{\partial x^{2}}+\frac{\partial^{2} u}{\partial y^{2}}+\frac{\partial^{2} u}{\partial z^{2}}\right)=\frac{1}{\varphi} \frac{\partial u}{\partial t}
$$

With the use of Taylor series expansion and by neglecting higher-order terms, the first term on the left-hand side in equation(23) can be expressed as equation (24) which is 2 nd order accuracy.

$$
\left(\frac{\partial^{2} u}{\partial x^{2}}\right)_{x, y, z}=\frac{u(x+\Delta x, y, z)-2 u(\mathrm{x}, \mathrm{y}, \mathrm{z})+u(x-\Delta x, y, z)}{(\Delta x)^{2}}
$$

In a similar manner, the second term and third term of the left-hand side in the equation(23) can be expressed as

$$
\begin{aligned}
& \left(\frac{\partial^{2} u}{\partial y^{2}}\right)_{x, y, z}=\frac{u(x, y+\Delta y, z)-2 u(\mathrm{x}, \mathrm{y}, \mathrm{z})+u(x, y-\Delta y, z)}{(\Delta y)^{2}} \\
& \left(\frac{\partial^{2} u}{\partial z^{2}}\right)_{x, y, z}=\frac{u(x, y, z+\Delta z)-2 u(\mathrm{x}, \mathrm{y}, \mathrm{z})+u(x, y, z-\Delta z)}{(\Delta z)^{2}}
\end{aligned}
$$

Replacing $\mathrm{x}$ and $\mathrm{y}$ and $\mathrm{z}$ with indices $\mathrm{i}$ and $\mathrm{j}$ and $\mathrm{k}$ the equations (24-26) and central difference scheme, the following equations can be obtained as shown in equations(27-29).

$$
\left(\frac{\partial^{2} u}{\partial x^{2}}\right)_{i, j, k}=\frac{u(i+1, j, k)-2 u(i, j, k)+u(i-1, j, k)}{(\Delta x)^{2}}
$$




$$
\begin{aligned}
& \left(\frac{\partial^{2} u}{\partial y^{2}}\right)_{i, j, k}=\frac{u(i, j+1, k)-2 u(i, j, k)+u(i, j-1, k)}{(\Delta y)^{2}} \\
& \left(\frac{\partial^{2} u}{\partial z^{2}}\right)_{i, j, k}=\frac{u(i, j, k+1)-2 u(i, j, k)+u(i, j, k-1)}{(\Delta z)^{2}}
\end{aligned}
$$

In a similar manner, using a forward time scheme, the right-hand side in the equation (23) can be expressed as

$$
\left(\frac{1}{\varphi} \frac{\partial u}{\partial t}\right)_{x, y, z}=\left(\frac{1}{\varphi} \frac{\partial u}{\partial t}\right)_{i, j, k}=\frac{1}{\varphi} \frac{u_{i, j, k}^{m+1}-u_{i, j, k}^{m}}{\Delta t}
$$

Replacing equations(27-30) in equation(23), the resulting 3D transient governing heat equation takes in the form as shown in equation(31). The equation(31) is based on Forward Time Central Space (FTCS) scheme, which is most commonly used [36].

$$
\begin{aligned}
& \frac{u_{(i+1, j, k)}-2 u_{(i, j, k)}+u_{(i-1, j, k)}}{(\Delta x)^{2}}+\frac{u_{(i, j+1, k)}-2 u_{(i, j, k)}+u_{(i, j-1, k)}}{(\Delta y)^{2}} \\
& +\frac{u_{(i, j, k+1)}-2 u_{(i, j, k)}+u_{(i, j, k-1)}}{(\Delta z)^{2}}=\frac{1}{\varphi} \frac{u_{i, j, k}^{m+1}-u_{i, j, k}^{m}}{\Delta t}
\end{aligned}
$$

In the present case, a parallelepiped of characteristic dimensions $0 \leq x \leq 1,0 \leq y \leq 1,0 \leq z \leq 1$ has been taken. The computational 3D mesh along with the stencil for the same is shown in Figure 2. One of the faces (yz) is kept at 100 degrees Celsius, and all other volume faces are kept at room temperature (30 degrees Celsius). The thermal diffusivity of the material under consideration is $\varphi=9.75 \times 10^{-6} \mathrm{~m}^{2} / \mathrm{s}$.

Determining the temperature field in position $u_{i, j, k}$ for any temporal value $\left(u^{m+1}\right)$, the solution of equation(31) is desired in all interior grid points. To obtain the stable and meaningful solution of equation (31), an optimal value of $\Delta t$ is to be taken depending on $\Delta x$, $\Delta y, \Delta z$ and based on the Fourier stability number $F_{u}$ [38]. As for the case of the 3D case [39],

$$
\left(\frac{\varphi \Delta t}{(\Delta x)^{2}}+\frac{\varphi \Delta t}{(\Delta y)^{2}}+\frac{\varphi \Delta t}{(\Delta z)^{2}}\right) \leq \frac{1}{2}
$$

Considering equal spacing of grid points along $\mathrm{x}, \mathrm{y}$ and $\mathrm{z}$ directions, $\Delta x=\Delta y=\Delta z=\Delta h$ in equation (32), the following relation can be obtained for stability criteria (equations $(33,34)$,

$$
\begin{aligned}
& F_{u} \leq \frac{1}{6} \\
& \text { where, } F_{u}=\varphi \frac{\Delta t}{\Delta h^{2}}
\end{aligned}
$$

\subsection{Assumptions for the analysis.}

In this present study, the assumptions taken have been given below:

1) Pure 3-dimensional heat conduction/diffusion

2) Cartesian equal grids along the direction of heat flow

3) Constant diffusive property of the material

4) No heat generation in the system

5) Effects of Radiation and advection on the transfer of heat have been neglected 

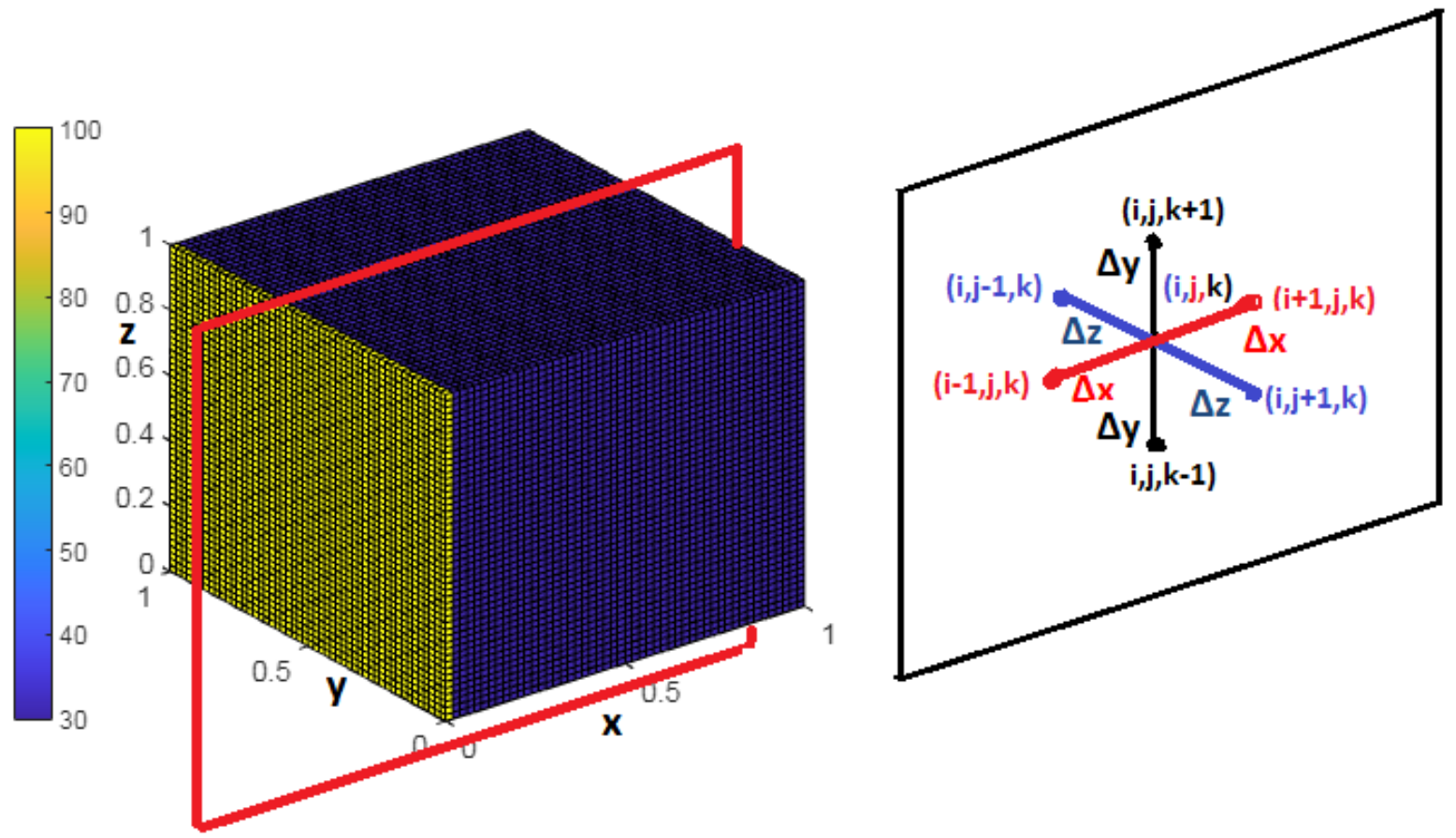

Figure 2. Schematic of 3D mesh with a computational stencil.

\begin{tabular}{|c|c|}
\hline $\begin{array}{l}\text { At } i=1, j=1, k=1, m=1 \\
u(2,1,1)+u(0,1,1)+u(1,2,1)+u(1,0,1)+u(1,1,2)+u(1,1,0)-6 u(1,1,1)-6 u(1,1,1,1)\end{array}$ & $+u(1,1,1,0)=0$ \\
\hline $\begin{array}{l}\text { At } i=2, j=1, k=1, m=1 \\
u(3,1,1)+u(1,1,1)+u(2,2,1)+u(2,0,1)+u(2,1,2)+u(2,1,0)-6 u(2,1,1)-6 u(2,1,1,1)\end{array}$ & $+u(2,1,1,0)=0$ \\
\hline $\begin{array}{l}\text { At } i=3, j=1, k=1, m=1 \\
u(4,1,1)+u(2,1,1)+u(3,2,1)+u(3,0,1)+u(3,1,2)+u(3,1,0)-6 u(3,1,1)-6 u(3,1,1,1)\end{array}$ & $+u(3,1,1,0)=0$ \\
\hline $\begin{array}{l}\text { At } i=1, j=2, k=1, m=1 \\
u(2,2,1)+u(0,2,1)+u(1,2,1)+u(1,1,1)+u(1,2,2)+u(1,2,0)-6 u(1,2,1)-6 u(1,2,1,2)\end{array}$ & $+u(1,2,1,1)=0$ \\
\hline $\begin{array}{l}\text { At } i=1, j=3, k=1, m=1 \\
u(2,3,1)+u(0,3,1)+u(1,4,1)+u(1,2,1)+u(1,3,2)+u(1,3,0)-6 u(1,3,1)-6 u(1,3,1,2)\end{array}$ & $+u(1,2,1,1)=0$ \\
\hline $\begin{array}{l}\text { At } i=1, j=1, k=2, m=1 \\
u(2,1,2)+u(0,1,2)+u(1,2,2)+u(1,0,2)+u(1,1,3)+u(1,1,1)-6 u(1,1,2)-6 u(1,1,2,2)\end{array}$ & $+\mathrm{u}(1,1,2,1)=0$ \\
\hline $\begin{array}{l}\text { At } i=1, j=1, k=3, m=1 \\
u(2,1,3)+u(0,1,3)+u(1,2,2)+u(1,0,2)+u(1,1,3)+u(1,1,1)-6 u(1,1,2)-6 u(1,1,2,2)\end{array}$ & $+\mathrm{u}(1,1,2,1)=0$ \\
\hline $\begin{array}{l}\text { At } i=2, j=2, k=2, m=1 \\
u(3,2,2)+u(1,2,2)+u(2,3,2)+u(2,1,2)+u(2,2,3)+u(2,2,1)-6 u(2,2,2)-6 u(2,2,2,2)\end{array}$ & $+u(2,2,2,1)=0$ \\
\hline $\begin{array}{l}\text { At } i=80, j=80, k=80, m=1 \\
u(81,80,80)+u(79,80,80)+u(80,81,80)+u(80,79,80)+u(80,80,81)+u(80,80,79) \\
-6 u(80,80,80)-6 u(80,80,80,2)+u(80,80,80,1)=0\end{array}$ & \\
\hline
\end{tabular}

Figure 3. Sample Linear Algebraic Equations (LAEs) derived from equation (31).

\subsection{Methodology.}

The solution of the FTCS equation (31) has been carried out for all space mesh points ( $\mathrm{i}=1, \mathrm{~N}_{\mathrm{x}}-1, \mathrm{j}=1, \mathrm{~N}_{\mathrm{y}}-1, \mathrm{k}=1, \mathrm{~N}_{\mathrm{z}}-1$ ) and for all time steps, where $\mathrm{N}_{\mathrm{x}}, \mathrm{N}_{\mathrm{y}}$, and $\mathrm{N}_{\mathrm{z}}$ are the total number of nodes along with the three orthogonal directions. To optimize the number of nodes for the numerical analysis, three different sets of node count have been chosen, which are (82 x $82 \times 82),(102 \times 102 \times 102)$, and (122 x 122x 122). Equation (31) when applied to three 
different sets of internal node points, ( $80 \times 80$ x 80), (100 x $100 \times 100)$, and (120 x 120x 120). It resulted in $5.12 \times 10^{5}, 1.0 \times 10^{6}$, and $1.728 \times 10^{6}$ linear algebraic equations (LAEs) for the particular instant of time, and they are illustrated in Figure 3. These LAEs (Figure 3) are solved using the popular iterative explicit-numerical scheme Liebmann method with proper numerical stability conditions [40]. As stated earlier, an efficient parallelized code has been developed and written in the MATLAB (R-2021a) platform. As the code is quite exhaustive, a screenshot of a portion of the code has been provided in Figure 4 so that one can have an idea of it. The validation of the code is being done by comparing the results obtained from the equation (21) with the iterative numerical scheme, and the results show the maximum error of $0.1 \%$ against the convergence $\left(10^{-6}\right)$. As discussed earlier, the grid dependency test has also been carried out using three different sets of nodes, and no difference in the results was observed. Hence, $(82 \mathrm{x}$ $82 \times 82$ ) nodes were selected for all the numerical experimentation.

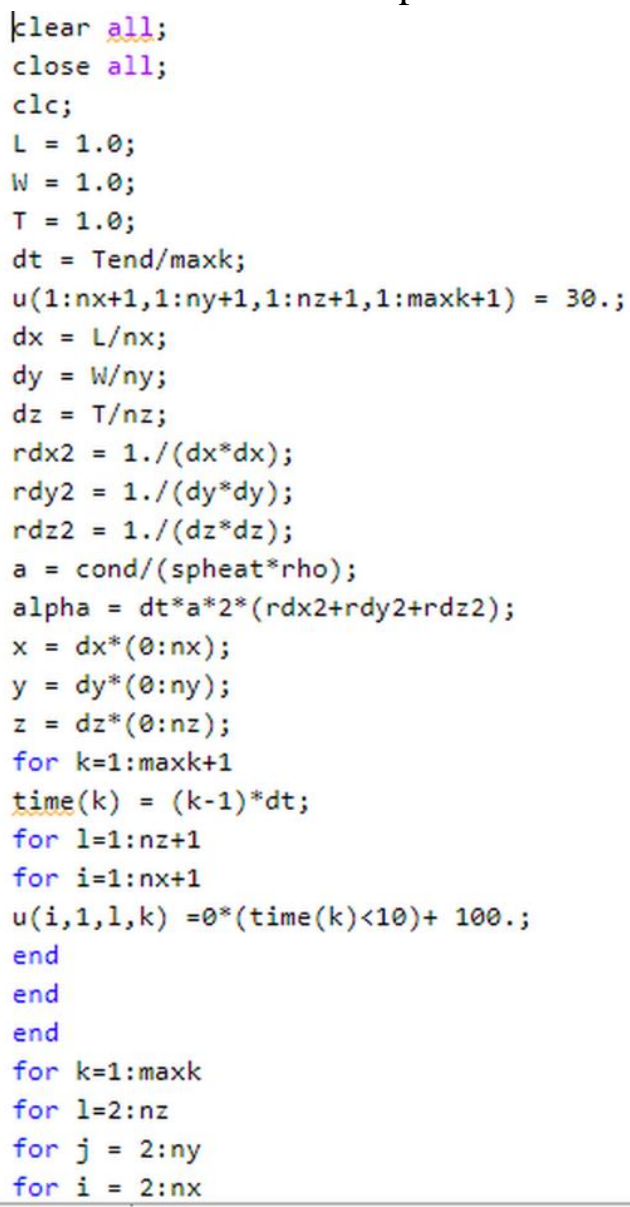

Figure 4. Snapshot of the developed code written in MATLAB (R-2021a) platform under vectorized cloud computing.

\section{Results and Discussion}

Using the assumptions stated above and the various equations and boundary conditions, the code was developed and run in MATLAB platform under vectorized cloud computing. The results were provided, and 3D plots were generated. After many iterations and refinement, the results were found to be consistent and stored. For better understanding and visualization, the visual results presented here are categorized under two sections:

a) Iso-contours at a particular temperature at different time steps

b) Iso-contour for different temperatures at a particular time step 
Figure 5 is for case (a), whereas Figure 6 caters to case (b). The choice of temperature (in case (a)) and choice of step (in case (b)) are taken arbitrarily as the contour is similar in all the cases; only the value changes. For better visualization and understanding of the complexity of the solution, Figure 7 has been provided to show the temperature contour and corresponding iso-contours at the $500^{\text {th }}$ time step.

On closely observing the figures, it can be inferred that the temperature gradient is initially very high, which gets smoothened subsequently. So it clearly indicates that in the initial machining period, a high amount of heat gets generated locally, resulting in thermal stresses being stored in the insert, which can be the major constraint for dimensional or even structural stability. Hence proper cooling and heat removal strategy is required to be implemented for such critical machined components.

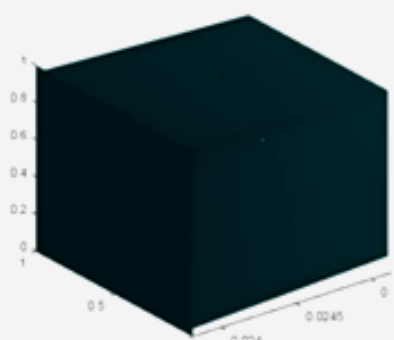

(a)

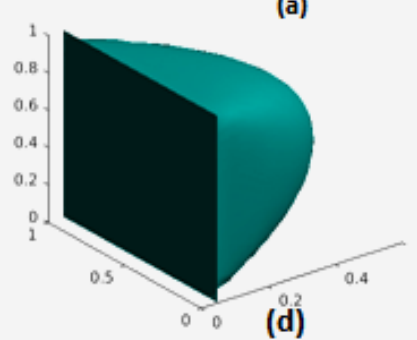

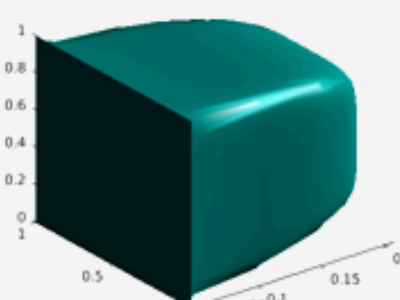

(b)

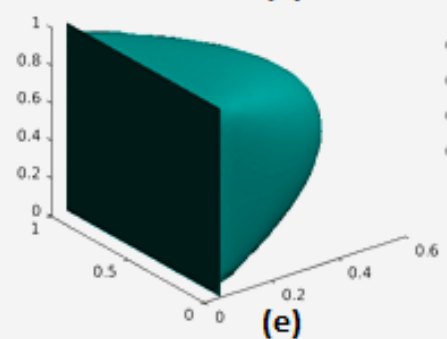

(e)

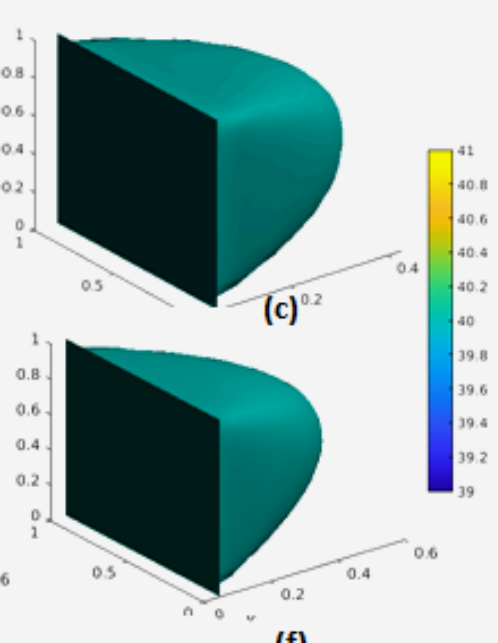

(f)

Figure 5. Iso-contours for $40 \mathrm{deg} C$ temperature at different time step: (a) Initial; (b) $50^{\text {th }}$ time step; (c) $100^{\text {th }}$ time step; (d) $150^{\text {th }}$ time step; (e) $200^{\text {th }}$ time step; (f) $500^{\text {th }}$ time step.

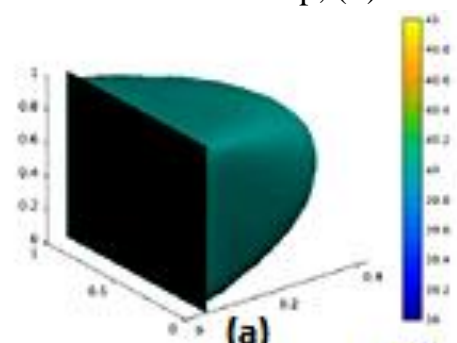

(a)

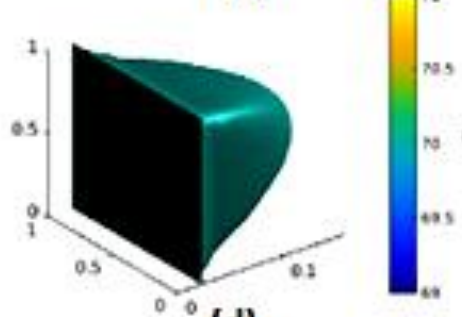

(d)

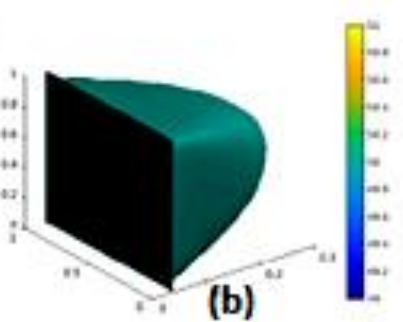

(b)
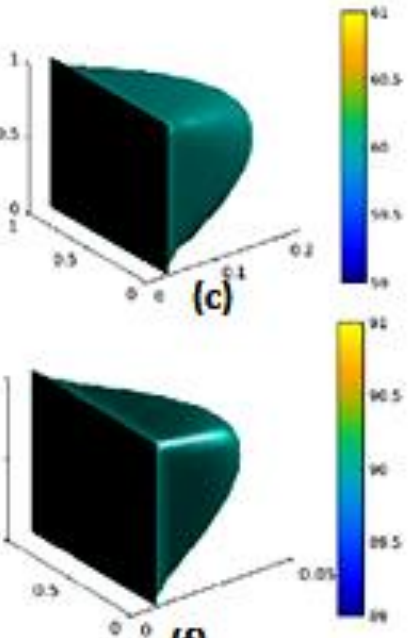

(f)

Figure 6. Iso-contour for different temperatures at 50th time step: (a) $40 \mathrm{deg} C$; (b) $50 \mathrm{deg}$ C; (c) $60 \mathrm{deg}$ C; (d) $70 \operatorname{deg}$ C; (e) $80 \operatorname{deg}$ C; (f) $90 \operatorname{deg}$ C. 


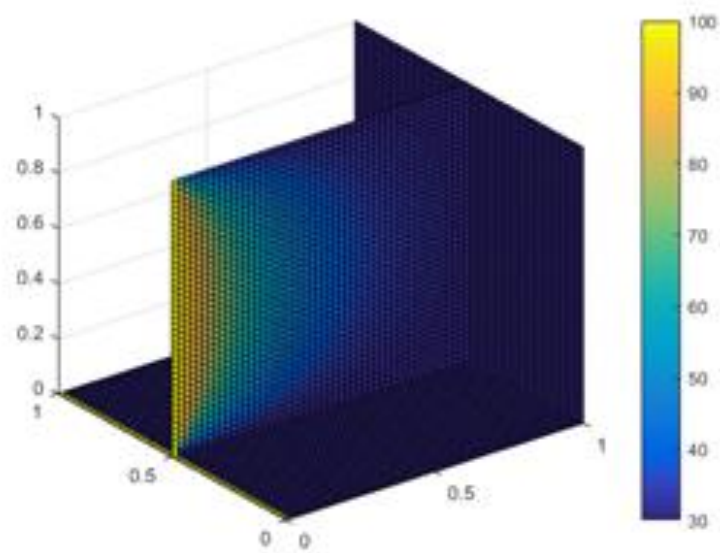

Figure 7. Temperature contour and corresponding iso-contours at $500^{\text {th }}$ time step.

\section{Conclusions}

Dental implants have become quite common today, and the most widely used material is titanium-based alloy like Ti-6Al-4V, which suffers difficulty in processing and machining due to its thermo-physical properties. Due to its hardness and difficult to machine characteristics, while machining, a large amount of heat gets generated, creating dimensional and even structural errors. The present work is directed towards investigating the temperature gradient and its profile so that before the assembly of the two parts, they are to be processed for stress relieving, and also a concrete cooling strategy is also required to reduce the thermal stresses in situ. The study was done with the above objective and provided information on the thermal field and distribution of temperature in the material during various machining stages. Earlier people $[23,24]$ have done similar work under a 2D environment, simpler than a 3D one but at the cost of incomplete information. This work was done with a 3D environment and was possible only with Vectorized Cloud Computing due to its complexity. The work resulted in the following generalized outcomes:

During machining of such materials, the temperature gradient is very high initially, which gets smoothened subsequently. So in the initial machining period, a high amount of heat generation results in developing a large amount of local thermal stresses, causing dimensional or even structural instability. Hence initially, the machining time has to be increased with proper choice of machining parameters.

As an augmentation to the above proper cooling and heat removal strategy must also be implemented to arrest the temperature gradient further and remove heat generated.

The various data generated by this work would help the designer, manufacturer, and all the related personnel for accurate, compatible and sustainable implants for the patients. Last but never least, society would get a better product to use.

\section{Funding}

This research received no external funding.

\section{Acknowledgments}

None. 


\section{Conflicts of Interest}

The authors declare no conflict of interest.

\section{References}

1. Agrawal, C.; Khanna, N.; Gupta, M. K.; Kaynak, Y. Sustainability assessment of in-house developed environment-friendly hybrid techniques for turning Ti-6Al-4V, Sustainable Materials and Technologies, Volume 26, 2020, e00220, https://doi.org/10.1016/j.susmat.2020.e00220.

2. Dhal, A. K.; Panda, A.; Kumar, R.; Sahoo, A. K.; Different machining environments impact analysis for Ti6Al-4V alloy (Grade 5) turning process: A scoping review, Materials Today: Proceedings, Volume 44, Part 1, 2021, Pages 2342-2347, https://doi.org/10.1016/j.matpr.2020.12.432.

3. Bowmanville dental. What to expect during dental implant treatment. Available online: https://bowmanvilledental.com/dental-implants-bowmanville/what-to-expect/ (accessed on August 9, 2021).

4. Markarian, R. A.; Galles, D. P.; França, F. M.G. Dental implant-abutment fracture resistance and wear induced by single-unit screw-retained CAD components fabricated by four CAM methods after mechanical cycling, The Journal of Prosthetic Dentistry, 2021, https://doi.org/10.1016/j.prosdent.2020.08.052.

5. Mruthunjaya, M.; Yogesha, K.B. A review on conventional and thermal assisted machining of titanium based alloy, I, 2021, https://doi.org/10.1016/j.matpr.2021.03.490.

6. Southern Implants. Machined Surface Coronal (MSc) Dental Implants. Available online: https://southernimplants.com/solutions/innovative-products/msc-implants/ (accessed on August 9, 2021).

7. Turkyilmazoglu, M. Numerical and Analytical Solutions for the Flow and Heat Transfer Near the Equator of an MHD Boundary Layer over a Porous Rotating Sphere. Int. J. Therm. Sci. 2011, 50, 831-842, https://doi.org/10.1016/j.ijthermalsci.2010.12.014.

8. Liang, C.; Zhao, J.F.; Chang, J.; Wang, H.P. Microstructure evolution and nano-hardness modulation of rapidly solidified $\mathrm{Ti}-\mathrm{Al}-\mathrm{Nb}$ alloy, Journal of Alloys and Compounds 2020, 836, 155538, https://doi.org/10.1016/j.jallcom.2020.155538.

9. Khanna, N.; Shah, P.; López de Lacalle, L. N.; Rodríguez, A.; Pereira, O. In pursuit of sustainable cutting fluid strategy for machining Ti-6Al-4V using life cycle analysis, Sustainable Materials and Technologies 2021, 29, e00301, https://doi.org/10.1016/j.susmat.2021.e00301.

10. Thirumaleshwar, M. Fundamentals of Heat and Mass Transfer (Pearson Education India, 2009).

11. Aghaee-Shalmani, Y,; Hakimzadeh, H. Numerical Modeling of 2-D and 3-D Flows using Artificial Compressibility Method and Collocated Mesh. J. Appl. Fluid Mech. 2016, 9, 2333-2345, https://doi:10.18869/acadpub.jafm.68.236.21800.

12. Karaa, S.; Zhang, J. Comput. Math. Appl. 2002, 44, 457-479, https://doi.org/10.1016/S0898-1221(02)001621.

13. Mishra, T. N. Analytical Solution of 2D SPL Heat Conduction Model, Int. j. latest res. 2015, 1, 47-54.

14. Kumar, S.; Khan, M.A,; Muralidharan, B. Processing of Titanium-Based Human Implant Material using Wire EDM. Mater. Manuf. Process. 2019, 34, 695-700, https://doi.org/10.1080/10426914.2019.1566609.

15. Shen, S.; Dai, W.; Cheng, J. Fractional parabolic two-step model and its accurate numerical scheme for nanoscale heat conduction, Journal of Computational and Applied Mathematics 2020, 375, 112812, https://doi.org/10.1016/j.cam.2020.112812.

16. Iqbal, M.; Stark, D.; Gimperlein, H.; Mohamed, M.S.; Laghrouche, O. Local adaptive q-enrichments and generalized finite elements for transient heat diffusion problems, Computer Methods in Applied Mechanics and Engineering 2020, 372, 113359, https://doi.org/10.1016/j.cma.2020.113359.

17. Lewis, B. J.; Onder, E. N.; Prudil, A. A. Chapter 6 - Difference numerical methods, Editor(s): Brent J. Lewis, E. Nihan Onder, Andrew A. Prudil, Advanced Mathematics for Engineering Students, ButterworthHeinemann 2022, 165-188, https://doi.org/10.1016/B978-0-12-823681-9.00014-9.

18. Gu, Y.; Fan, C. M.; Qu, W. Z.; Wang, F. Localized method of fundamental solutions for large-scale modelling of three-dimensional anisotropic heat conduction problems - Theory and MATLAB code, Computers \& Structures 2019, 220, 144-155, https://doi.org/10.1016/j.compstruc.2019.04.010.

19. Kovtanyuk, A.E.; Chebotarev A.Y. An Iterative Method for Solving a Complex Heat Transfer Problem. Appl. Math. Comput. 2013, 219, 9356-9362, https://doi.org/10.1016/j.amc.2013.03.091. 
20. Lam, T.T.; Fong, E. Application of Solution Structure Theorem to Non-Fourier Heat Conduction Problems: Analytical Approach, Int. J. Heat Mass Transf. 2011, 54, 4796-4806, https://doi.org/10.1016/j.ijheatmasstransfer.2011.06.028.

21. Lam, T.T.; A Unified Solution of Several Heat Conduction Models, Int. J. Heat Mass Transf, 2013, 56, 653666, https://doi.org/10.1016/j.ijheatmasstransfer.2012.08.055.

22. Woodbury, K.A.; Najafi, H,; Beck, J.V. Exact Analytical Solution for 2-D Transient Heat Conduction in a Rectangle with Partial Heating on One Edge, Int. J. Therm. Sci. 2017, 112, 252-262, https://doi.org/10.1016/j.ijthermalsci.2016.10.014.

23. Miranda, G. H. B. ; Felipe, J. C. Computer-Aided Diagnosis System Based on Fuzzy Logic for Breast Cancer Categorization. Comput. Biol. Med. 2015, 64, 334-346, https://doi.org/10.1016/j.compbiomed.2014.10.006.

24. Frunzā, M. F.; Luca, R. The use of Numerical Applications in the Study of Dental Contacts, Appl. Med. Inform 2010, 26, 73-82, https://ami.info.umfcluj.ro/index.php/AMI/article/view/36.

25. Bahşi, M. M.; Çevik, M. Taylor Matrix Solution of the Mathematical Model of the RLC Circuits, Math. Comput. Appl. 2013, 18, 467-475, https://doi.org/10.3390/mca18030467.

26. Wang, S,; Ni, R. Solving of Two-Dimensional Unsteady-State Heat-Transfer Inverse Problem Using Finite Difference Method and Model Prediction Control Method, Complexity 2019, 2019, 1-12, https://doi.org/10.1155/2019/7432138.

27. Koh, Y. Y.; Lim, J. W. S.; Chua, Y. L. Multigrid Solver for 2D Heat Conduction Problems, AIP Conference Proceedings. 2019, 2129, 020033-1-020033-6, https://doi.org/10.1063/1.5118041.

28. Nemirovsky, Y.V. ; Mozgova, A.S. Two-Dimensional Steady-State Heat Conduction Problem for Heat Networks, J. Phys.: Conf. Ser. 2019, 1359, 012138, 1-5, https://doi.org/10.1088/1742-6596/1359/1/012138.

29. Hu, Z.; Liu, Z. Heat Conduction Simulation of 2D Moving Heat Source Problems Using a Moving Mesh Method, Adv. Math. Phys. 2020, 6067854, 1-16, https://doi.org/10.1155/2020/6067854.

30. Fong, Ed,; Yi, Sonny,; Lam, T. T. Heating and Convective Cooling of Two-Dimensional Solids Using Diffusion and Cattaneo-Vernotte Models, J. Thermophys. Heat Transf. 2021, 1-12, https://arc.aiaa.org/doi/pdf/10.2514/1.T6255.

31. Udoye, N.E.; Okolie, S.T.A.; Fayomi, O.S.I.; Banjo, S.O. Computational analysis of conductive heat transfer in a rectangular slab of stable boundary using Monte Carlo method IOP Conf. Ser.: Mater. Sci. Eng. 2021, https://doi.org/10.1088/1757-899X/1036/1/012059.

32. Pranita, P.A.; Kumar, D. On Code Verification of 2D Transient Heat Conduction in Composite Wall, International Conference on Mechanical, Materials and Renewable Energy, IOP Conf. Series: Materials Science and Engineering 2018, 377, 012128, https://doi.org/10.1088/1757-899X/377/1/012128.

33. Roy, A.K.; Kumar, K. 2D heat conduction on a flat plate with Ti6Al4V alloy under steady state conduction: A numerical analysis, Materials Today: Proceedings, 46, 1, 2021, 896-902, https://doi.org/10.1016/j.matpr.2020.12.1152.

34. Roy, A.K.; Jeyapandiarajan, P.; Kumar, K. Numerical and Thermal Modelling of Machining Implants: A case with Ti6Al4V alloy with unsteady heat diffusion, Materials Today: Proceedings. 2021, https://doi.org/10.1016/j.matpr.2021.02.145.

35. Özisik, M.N.; Heat Conduction 1993, 2nd ed.; Wiley, New York.

36. Sharma, A. Introduction to Computational Fluid Dynamics: Development, Application and Analysis 2017, John Wiley and Sons Ltd., https://doi.org/10.1002/9781119369189.

37. Majumdar, M.; Solution of three dimensional heat equation (Maths), e-Pathsala, MHRD, GOI, https://www.youtube.com/watch?v=xkk6QW4FyZc\&list=RDCMUCCUr096WDp86n62CXBeHlQw\&inde $\mathrm{x}=1$.

38. Incropera, F.P.; Dewitt, D. P. 1996. Fundamentals of Heat and Mass Transfer (4th ed.), Wiley, New York.

39. Özişik, M.N.; Orlande, H.R.B.; Colaço, M.J.; Cotta, R.M. 2017. Finite Difference Methods in Heat Transfer (2nd ed.), CRC Press, https://doi.org/10.1201/9781315121475.

40. Langtangen, H.P.; Linge, S. Finite Difference Computing with PDEs: A Modern Software Approach, 2017, Springer Open (edited Book), https://doi.org/10.1007/978-3-319-55456-3. 